\title{
WASP-24 b: A NEW TRANSITING CLOSE-IN HOT JUPITER ORBITING A LATE F-STAR
}

\author{
R. A. Street ${ }^{1}$, E. Simpson ${ }^{2}$, S. C. C. Barros², D. Pollacco ${ }^{2}$, Y. Joshi², I. TodD ${ }^{2}$, A. Collier Cameron ${ }^{3}$, B. Enoch ${ }^{3}$,

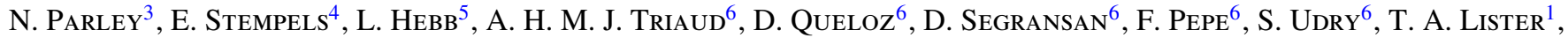 \\ É. Depagne ${ }^{1}$, R. G. West $^{7}$, A. J. Norton ${ }^{8}$, B. Smalley ${ }^{9}$, C. Hellier ${ }^{9}$, D. R. Anderson ${ }^{9}$, P. F. L. Maxted ${ }^{9}$, S. J. Bentley ${ }^{9}$,

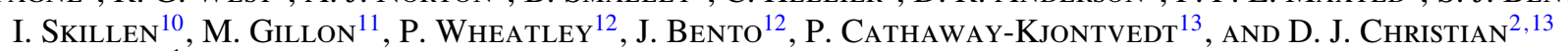 \\ ${ }^{1}$ Las Cumbres Observatory Global Telescope Network, 6740 Cortona Drive, Suite 102, Goleta, CA 93117, USA \\ 2 Astrophysics Research Centre, Physics Building, Queen's University, Belfast, County Antrim, BT7 1NN, UK \\ ${ }^{3}$ SUPA, School of Physics and Astronomy, University of St. Andrews, North Haugh, St. Andrews, KY16 9SS, UK \\ ${ }^{4}$ Department of Physics and Astronomy, P.O. Box 516, SE-751 20 Uppsala, Sweden \\ ${ }^{5}$ Physics \& Astronomy Department, Vanderbilt University, 1807 Station B, Nashville, TN 37235, USA \\ ${ }^{6}$ Observatoire Astronomique de l'Université de Genève, 51 Chemin des Maillettes, 1290 Sauverny, Switzerland \\ ${ }^{7}$ Department of Physics \& Astronomy, University of Leicester, University Road, Leicester, LE1 7RH, UK \\ ${ }^{8}$ Department of Physics \& Astronomy, The Open University, N2041, Venables Building, Milton Keynes, MK7 6AA, UK \\ 9 Astrophysics Group, Keele University, Staffordshire, ST5 5BG, UK \\ ${ }^{10}$ Isaac Newton Group of Telescopes, Apartado de correos 321, E-38700 Santa Cruz de la Palma, Canary Islands, Spain \\ ${ }^{11}$ Institut d'Astrophysique et de Géophysique, Université de Liège, Allée du 6 Août 17, Bât. B5C, 4000 Liège, Belgium \\ ${ }^{12}$ Department of Physics, University of Warwick, Gibbet Hill Road, Coventry, CV4 7AL, UK \\ ${ }^{13}$ Department of Physics and Astronomy, California State University Northridge, 18111 Nordhoff Street, Northridge, CA 91330, USA \\ Received 2010 March 12; accepted 2010 July 3; published 2010 August 10
}

\begin{abstract}
We report the discovery of a new transiting close-in giant planet, WASP-24 b, in a 2.341 day orbit, 0.037 AU from its F8-9 type host star. By matching the star's spectrum with theoretical models, we infer an effective temperature $T_{\text {eff }}=6075 \pm 100 \mathrm{~K}$ and a surface gravity of $\log g=4.15 \pm 0.10$. A comparison of these parameters with theoretical isochrones and evolutionary mass tracks places only weak constraints on the age of the host star, which we estimate to be $3.8_{-1.2}^{+1.3}$ Gyr. The planetary nature of the companion was confirmed by radial velocity measurements and additional photometric observations. These data were fit simultaneously in order to determine the most probable parameter set for the system, from which we infer a planetary mass of $1.071_{-0.038}^{+0.036} M_{\mathrm{Jup}}$ and radius $1.3_{-0.037}^{+0.039} R_{\mathrm{Jup}}$.
\end{abstract}

Key words: planetary systems

Online-only material: color figures

\section{INTRODUCTION}

Large-scale, ground-based surveys for transiting planets are yielding a surprisingly diverse set of close-in giant planets. The last few years have seen the discovery of a number of socalled bloated close-in Jovian planets, for example, WASP-17 b (Anderson et al. 2010) and Kepler-7 b (Latham et al. 2010). The very low densities of these objects present an ongoing challenge to theories of planet formation and evolution (Fortney et al. 2008; Guillot et al. 2006; Burrows et al. 2007). Ultrashort period planets such as WASP-19 b (Hebb et al. 2010) offer a testbed for the physics of the dissipation of tidal energy, thought to both bolster the planetary radius (Fortney et al. 2007; Burrows et al. 2007) and perhaps cause the planet's orbits to decay (Jackson et al. 2009), ultimately leading them to spiral into their host stars. Each new wave of planets discovered has produced new surprises. As the surveys searching for new systems reach maturity, and are complemented by targeted space-based missions, we are populating a wider range of the planetary orbital and physical parameter space. A more complete picture of the planetary menagerie will lead to a better understanding of the formation and evolutionary forces at work.

There is a particular value in completing a census of the transiting planets of bright stars, which is evident from the extraordinary insights offered by follow-up work into the composition, structure, and even weather of their atmospheres and exospheres (e.g., Désert et al. 2009; Knutson et al. 2009; Burrows et al. 2009, and references therein). We may even be able to detect changes in weather patterns over the course of an orbit for the long period and eccentric planets (Iro \& Deming 2010). Ground-based instruments survey large numbers of bright stars spanning spectral types $\mathrm{F}-\mathrm{M}$ and produce targets well suited for further study.

We report the discovery of a new close-in giant planet orbiting an $11.3 \mathrm{mag}$, F8-9 type host star. Our observations are described in Section 2, including both our discovery data and follow-up work. In Section 3, we present the fitting procedure from which we determine the overall system parameters. We discuss the new system in the context of the current sample of known planets in Section 4.

\section{OBSERVATIONS}

\subsection{SuperWASP Discovery Data}

The WASP Consortium ${ }^{14}$ operates two fully robotic, dedicated observatories: WASP-North, sited at the Observatorio del Roque de los Muchachos (ORM), La Palma, Canary Islands, Spain, and WASP-South, hosted by the South African Astronomical Observatory at Sutherland, South Africa. Both stations support eight cameras, each consisting of a Canon $200 \mathrm{~mm}$, $\mathrm{f} / 1.8$ lens, and an Andor $2048 \times 2048$ pixel thinned e2v CCD. Each camera has a $7.8 \times 7.8$ field of view and a pixel scale of $13^{\prime \prime} .7$ pixel $^{-1}$. Every clear night the stations execute pre-set observing programs, repeatedly imaging a sequence of 6-12 planet fields every $\sim 8$ minutes for as long as they are visible. Full details of the hardware and data reduction procedures are

\footnotetext{
14 www.superwasp.org
} 
Table 1

Radial Velocity Observations of WASP-24

\begin{tabular}{lccccc}
\hline \hline $\begin{array}{c}\text { NOT Data } \\
\text { HJD-2,450,000 }\end{array}$ & $\begin{array}{c}\text { RV } \\
\left(\mathrm{km} \mathrm{s}^{-1}\right)\end{array}$ & $\begin{array}{c}\text { RV Error } \\
\left(\mathrm{km} \mathrm{s}^{-1}\right)\end{array}$ & $\begin{array}{c}\text { CORALIE Data } \\
\text { BJD-2,450,000 }\end{array}$ & $\begin{array}{c}\text { RV } \\
\left(\mathrm{km} \mathrm{s}^{-1}\right)\end{array}$ & $\begin{array}{c}\text { RV Error } \\
\left(\mathrm{km} \mathrm{s}^{-1}\right)\end{array}$ \\
\hline 4832.7716 & -17.745 & 0.012 & 4860.8807 & -17.652 & 0.022 \\
4833.8017 & -18.054 & 0.014 & 4894.8495 & -17.916 & 0.022 \\
4834.7794 & -17.735 & 0.015 & 4895.8482 & -17.647 & 0.015 \\
4835.7779 & -17.989 & 0.028 & 4896.8643 & -17.937 & 0.018 \\
4836.7888 & -17.902 & 0.018 & 4897.8061 & -17.731 & 0.016 \\
4862.7251 & -17.778 & 0.022 & 4898.8552 & -17.841 & 0.018 \\
4910.6468 & -18.008 & 0.012 & 4941.7513 & -17.912 & 0.015 \\
4922.6956 & -18.105 & 0.018 & 4942.7986 & -17.691 & 0.018 \\
4931.6231 & -17.877 & 0.030 & 4943.7765 & -17.952 & 0.018 \\
4932.5743 & -17.967 & 0.019 & 4944.7651 & -17.701 & 0.017 \\
& & & 4946.7581 & -17.815 & 0.015 \\
& & & 4947.7215 & -17.729 & 0.015 \\
& & 4948.6961 & -17.961 & 0.014 \\
& & 4950.7990 & -17.915 & 0.017 \\
& & 4972.7427 & -17.718 & 0.018 \\
& & 4985.6711 & -17.891 & 0.015 \\
& & 5013.5912 & -17.803 & 0.020 \\
& & 5038.5044 & -17.675 & 0.017 \\
\hline
\end{tabular}

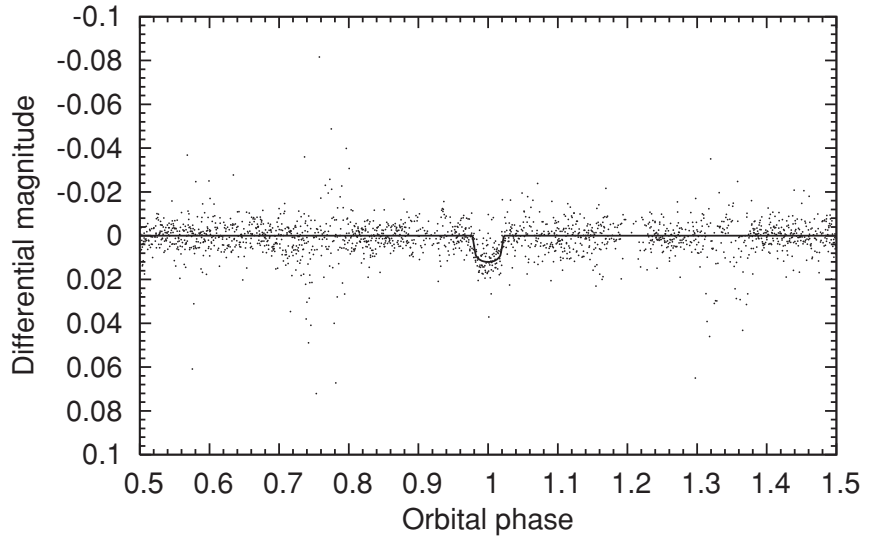

Figure 1. Combined WASP-North and WASP-South light curve for WASP-24, folded on the ephemeris derived from this and follow-up data sets and averaged in bins of width $120 \mathrm{~s}$. Superimposed is the model transit light curve, based on the determined system parameters.

given in Pollacco et al. (2006). Collier Cameron et al. (2006) describe the HUNTSMAN algorithm used to search the data for transits.

WASP-North and WASP-South have been observing WASP-24 since 2008 March 5, accumulating a light curve of 9750 data points by 2009 April 28, shown in Figure 1. Once flagged by HUNTSMAN, the WASP light curve for this star was manually evaluated along with all available catalog data (for a description of our selection procedure, see Collier Cameron et al. 2007), and the object was put forward for follow-up.

\subsection{Spectroscopic Follow-up}

The $2.56 \mathrm{~m}$ Nordic Optical Telescope, sited at ORM, La Palma, provided the first radial velocity (RV) measurements of the WASP-24 system. The Fibre-Fed Echelle Spectrograph (FIES) was used in medium resolution mode $(R=46,000)$ with simultaneous ThAr wavelength calibration, giving spectra covering the wavelength range $370-720 \mathrm{~nm}$. A total of 10 spectroscopic observations were made between 2008 December 31 and 2009 April 10 with an exposure time of 1200 s. The data were reduced using the bespoke package
FIEStool and an IDL cross-correlation routine was used to derive the RVs.

Additional RV measurements were obtained between 2009 January 29 and 2009 July 26 with the CORALIE Fibre-Fed Echelle Spectrograph on the Swiss $1.2 \mathrm{~m}$ Leonard Euler Telescope at ESO-La Silla, Chile. These spectra span the wavelength range $390-690 \mathrm{~nm}$ with a resolution $\sim 50,000$. The RV measurements derived from both data sets are given in Table 1, and plotted in Figure 2.

These spectra showed no sign of the broadened lines which would indicate a rapidly rotating host star and make any planetary companion difficult to confirm. Nor did it exhibit the dual lines of a spectroscopic binary. The semiamplitude of variation was only $0.1452_{-0.0044}^{+0.0045} \mathrm{~km} \mathrm{~s}^{-1}$, indicating a companion of planetary mass. To exclude the possibility that this signal was caused by stellar activity, span bisector analysis was performed (Queloz et al. 2001) using the CORALIE data. The uncertainty in the bisector span was taken to be twice the formal error on the RV measurement, which is derived from the signalto-noise ratio of the spectrum and the contrast and FWHM of the cross-correlation function. Experience indicates that the rms scatter in the bisector span is approximately twice the rms of the RV residuals. Figure 2 (lower panel) shows that no significant variation was seen. In addition, these data would be expected to display a gradient if the RV signal was caused by a massive companion such as a brown dwarf orbiting one star in an unresolved binary (Santos et al. 2002). As no slope was seen, we concluded that the signal is due to an orbiting planetary companion. The star was therefore promoted in the target list for higher resolution imaging and photometry.

\subsection{Photometric Follow-up}

In order to better constrain the morphology of the light curve, we obtained photometry of additional transit events, as shown in Figure 3. The first complete transit was observed with the Liverpool Telescope using the RISE instrument on La Palma, Canary Islands on 2009 April 23. This camera consists of an e2v CCD47-20 frame transfer CCD with a field of view of $9.2 \times$ 9.2 and has a fixed broadband filter equivalent to "V+R." 

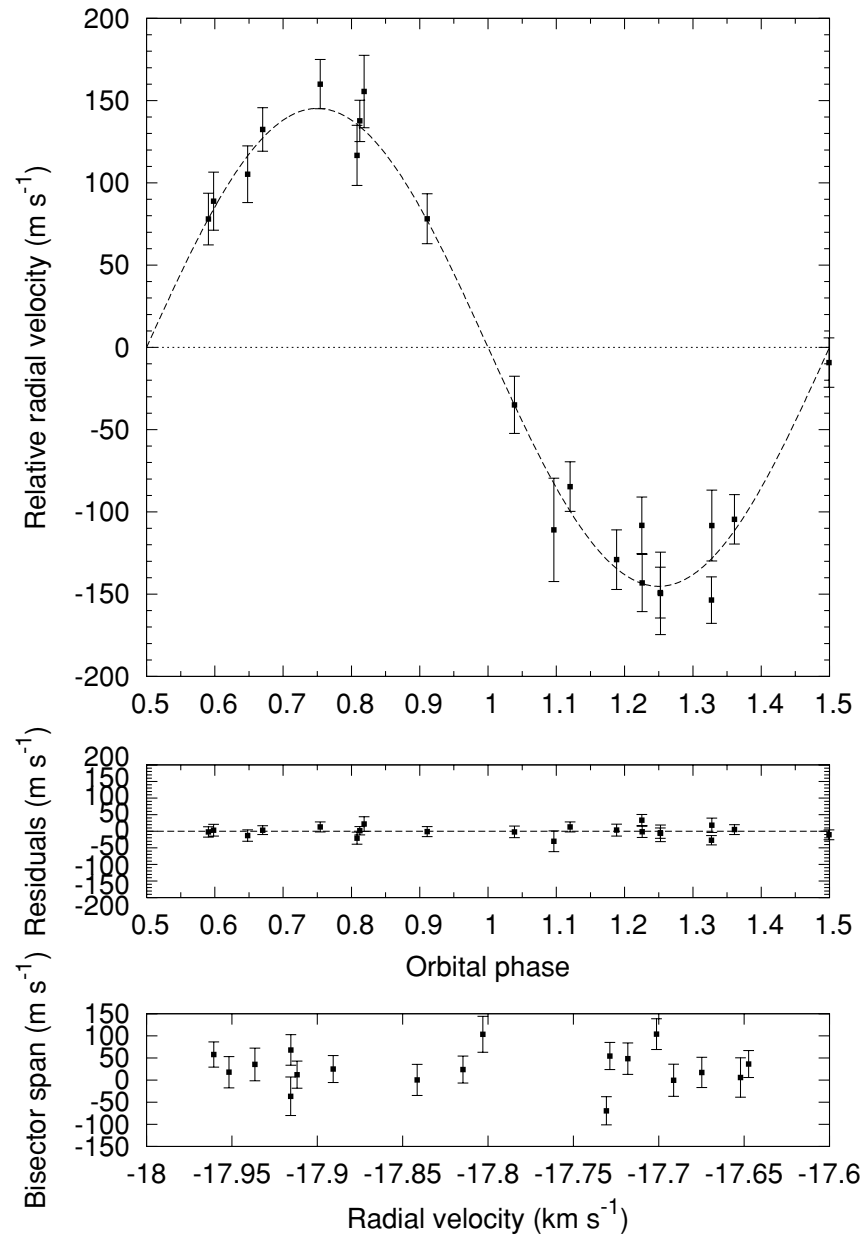

Figure 2. Upper panel: phase-folded RV measurements of WASP-24, combining data from the Swiss $1.2 \mathrm{~m} /$ CORALIE and the $2.56 \mathrm{~m}$ NOT/FIES. Middle panel: the RV residuals once the model for the orbital motion of the planet has been subtracted. Lower panel: the bisector span measurements from the coraliE data are plotted against their measured RV.

With a declination of $\sim+2^{\circ}$, WASP-24 is visible to both $2.0 \mathrm{~m}$ Faulkes Telescopes. Faulkes-North (FTN, Hawai'i) observed a near-complete transit on 2009 May 17 with the Merope camera plus the SDSS-i' filter, and a partial event on 2009 June 12. FTN and its southern counterpart Faulkes-South (FTS, Siding Spring, Australia) jointly observed the transit on 2009 July 17 using the Pan-STARRS-z filter. The Merope instruments were used at both telescopes for these events, and have similar properties. They contain an e2V, $2048 \times 2048$ pixel-CCD and have a field of view of $4^{\prime} .7 \times 4^{\prime} .7$. FTN observed this star again in 2010 on February 22, May 1, and May 15 and FTS caught another transit on 2010 April 26. This season, the Spectral camera was used with the Pan-STARRS-z filter because it offers a larger (10'3 $\times 10^{\prime} .2$ ) field of view and hence more comparison stars. The Spectral cameras contain $4096 \times 4096$ pixel Fairchild Imaging CCDs.

\subsection{Neighboring Eclipsing Binary}

In the course of obtaining photometric follow-up of WASP24 , we noticed that the nearest neighboring star (hereafter called N1; see Figure 4) showed deep ( $0.8 \mathrm{mag}$ ), "V"-shaped eclipses in a few, but not all, of our data sets (shown in Figure 5). This object, at $\alpha=150850.3, \delta=+022031.8$, has $V=$ $17.970 \mathrm{mag}$ (NOMAD Catalog) and is identified as USNO-B1.0

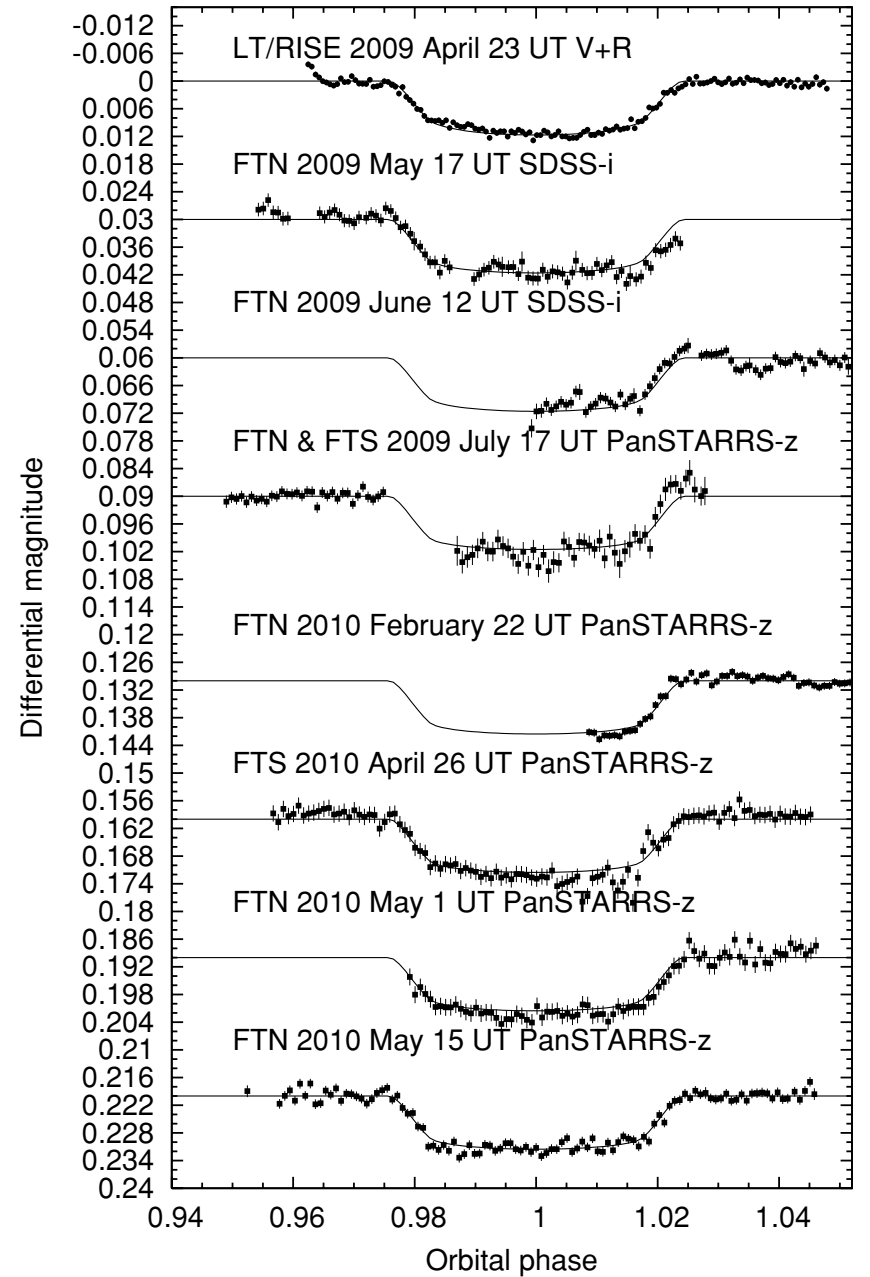

Figure 3. Photometry of transit events between 2009 April and 2010 May, offset in magnitude for clarity. The data have been binned to $120 \mathrm{~s}$. Superimposed are the model transit light curves based on the determined system parameters.

0923-0348082. It is present in the Two Micron All Sky Survey (2MASS) Point Source Catalog as 2MASS 15085031+0220313 with magnitudes $J=14.160 \mathrm{mag}, H=13.551 \mathrm{mag}$, and $K_{S}=$ 13.349 mag. Although these are the combined magnitudes of two stars, the colors imply a late $\mathrm{K} /$ early $\mathrm{M}$ spectral type.

This neighboring star lies $\sim 21^{\prime \prime}$. 2 from WASP-24. Although the two objects are blended in the SuperWASP imaging, N1 is $\sim 6.7$ mag fainter in V. N1 will have had minimal effect on the WASP transit photometry because even at its brightest it is below the limit of detectability in the SW exposures.

$\mathrm{N} 1$ is clearly separated from WASP-24 in the follow-up photometry, as shown in Figure 4, and was explicitly excluded from the list of comparison stars used to derive the differential photometry for the latter object. Eclipses of N1 were observed from both FTN and FTS, and the measured times of minima (Table 2) do not coincide with the transits of WASP-24. Additionally, they show a markedly different profile and amplitude. Finally, the neighbor is sufficiently separated from WASP-24 as to fall outside the spectrograph fiber during the CORALIE and NOT observations. From this, we conclude that the transits of WASP-24 are observed independently of the variability of its neighbor.

We used the Starlink package FROG (Allan 2004) to identify the period of this binary from the combined photometric data sets. This suggested a period of $P=1.156$ days but as the data sets do not cover the entire phase range, we cannot rule out integer multiples of this. We note that $2 P=2.312$ days, similar 

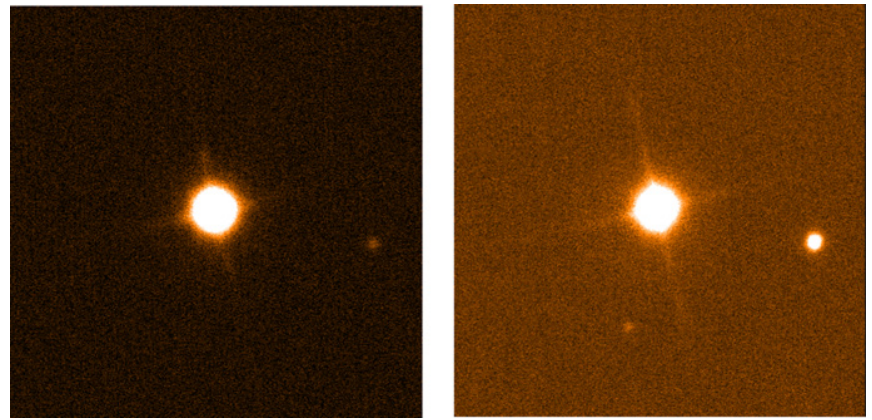

Figure 4. $1^{\prime} \times 1^{\prime}$ subframes from high-resolution (pixel scale $=0^{\prime}{ }^{\prime} 139$ pixel $^{-1}$ ) FTN imaging of WASP-24 in Bessel-B (left) and Pan-STARRS-z (right) passbands. North is up and east is left. The neighboring eclipsing binary is to the right of WASP-24 (center)

(A color version of this figure is available in the online journal.)

Table 2

Times of Eclipses of the Neighboring EB, Given in (HJD-2,450,000.0)

\begin{tabular}{ccc}
\hline \hline Measured $T_{0}$ & Date $(\mathrm{UT})$ & Telescope \\
\hline $5022.8319 \pm 0.0003$ & 2009 Jul 10 & FTN \\
$5023.9869 \pm 0.0002$ & 2009 Jul 11 & FTS \\
$5029.76805 \pm 0.00005$ & 2009 Jul 17 & FTN
\end{tabular}

to the period of WASP-24 ( $P=2.341$ days), but sufficiently different that our photometry from 2009 May-June, timed to observed transits of WASP-24, produced out of eclipse data for $\mathrm{N} 1$. The ephemerides of the two objects only produced minima on the same night in data from 2009 July.

Given the depth and profile of the eclipses of N1 and its very red overall color, it is likely to be a detached eclipsing binary of two late-type components.

\section{DERIVING SYSTEM PARAMETERS FOR WASP-24}

\subsection{Spectral Type}

We used the high-resolution NOT spectra to derive refined estimates for the stellar atmospheric parameters $T_{\text {eff }}$, surface gravity $\log g$, metallicity $[\mathrm{M} / \mathrm{H}]$, projected rotational velocity
Table 3

Basic Parameters of the Host Star, Including the Age and Mass as Derived in Section 3.3

\begin{tabular}{lc}
\hline \hline \multicolumn{1}{c}{ Parameter } & Value \\
\hline Identifiers & 1SWASP J150851.72+022036.1 \\
& USNO-B1.0 0923-0348089 \\
& 2MASS J15085174+0220358 \\
& TYCHO-2 339-329-1 \\
R.A. (J2000.0) & $15^{\mathrm{h}} 08^{\mathrm{m}} 51^{\mathrm{s} .72}$ \\
Decl. (J2000.0) & $+02^{\circ} 20^{\prime} 36^{\prime \prime} 1$ \\
$V_{\mathrm{SW}}$ & $11.306 \pm 0.108 \mathrm{mag}$ \\
$J$ & $10.457 \pm 0.022 \mathrm{mag}$ \\
$H$ & $10.219 \pm 0.026 \mathrm{mag}$ \\
$K_{s}$ & $10.148 \pm 0.023 \mathrm{mag}$ \\
$V_{\mathrm{SW}}-K_{S}$ & $1.079 \mathrm{mag}$ \\
$J-H$ & $0.238 \mathrm{mag}$ \\
Spectral type & $\mathrm{F}-9$ \\
$T_{\text {eff }}$ & $6075 \pm 100 \mathrm{~K}$ \\
$\log g$ & $4.15 \pm 0.10$ \\
{$[\mathrm{M} / \mathrm{H}]$} & $\pm 0.07+0.10$ \\
$v \sin i$ & $7.0 \pm 1.0 \mathrm{~km} \mathrm{~s}{ }^{-1}$ \\
$\log n(\mathrm{Li})$ & $2.42 \pm 0.1$ \\
Age & $3.8_{-1.2}^{+1.3} \mathrm{Gyr}$ \\
Mass & $1.15_{-0.04}^{+0.05} M_{\odot}$ \\
\hline
\end{tabular}

Notes. $J, H, K_{s}$ magnitudes are derived from the 2MASS catalog (Cutri et al. 2003). The quoted spectral type is derived from Gray (1992) based on the effective temperature.

$v \sin i$, and lithium abundance $\log n(\mathrm{Li})$. The NOT spectra were analyzed using the Spectroscopy Made Easy package (hereafter SME; Valenti \& Piskunov 1996) to fit a sequence of synthetic spectra. The best-fitting synthetic spectrum from this grid of models was then identified via a least-squares technique. Figure 6 overlays the NOT spectrum with the bestfitting synthetic model. For more details of this method, see Stempels et al. (2007). This analysis produced the estimates for the stellar atmospheric parameters given in Table 3.

The surface gravity, $\log g=4.15 \pm 0.1$, suggested that the star may have evolved away from the zero-age main sequence. This conclusion was supported by the low level of lithium (Sestito \&

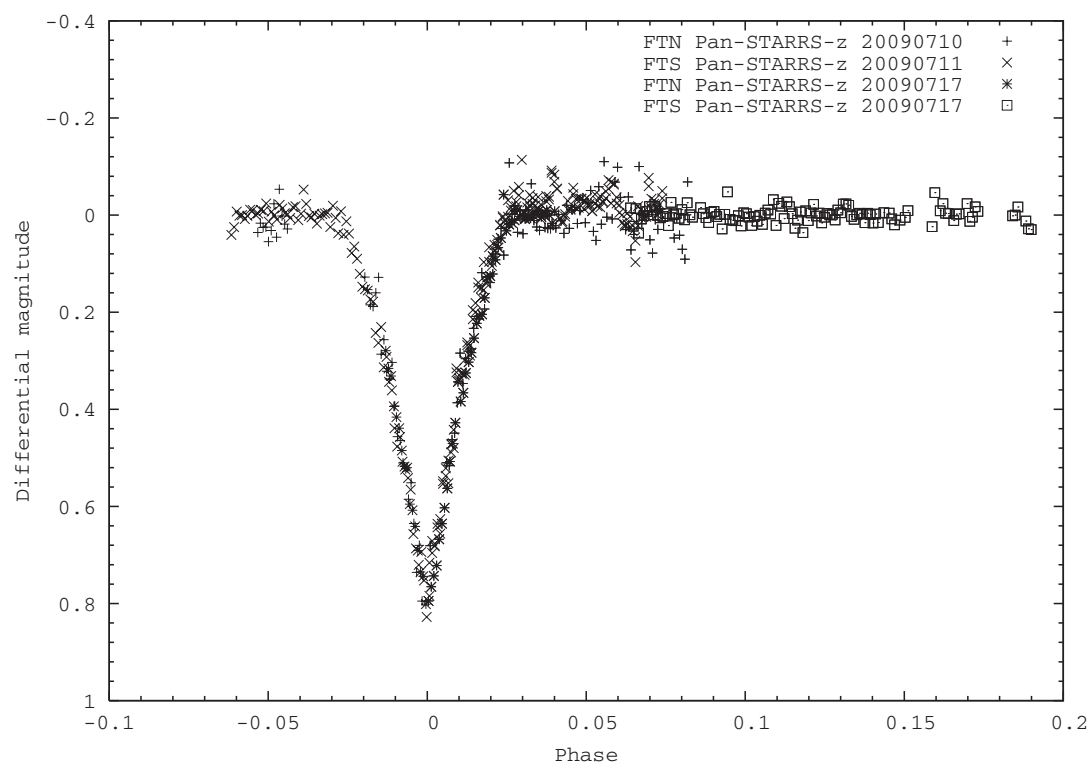

Figure 5. Light curve of the neighboring binary, folded on a period of $1.156 \mathrm{~d}$ and combining data from FTN and FTS in Pan-STARRS-z filter covering three eclipses. Other data sets in SDSS-i' and Bessell-I were obtained out of eclipse and were combined with the Pan-STARRS-z data to constrain the period. 

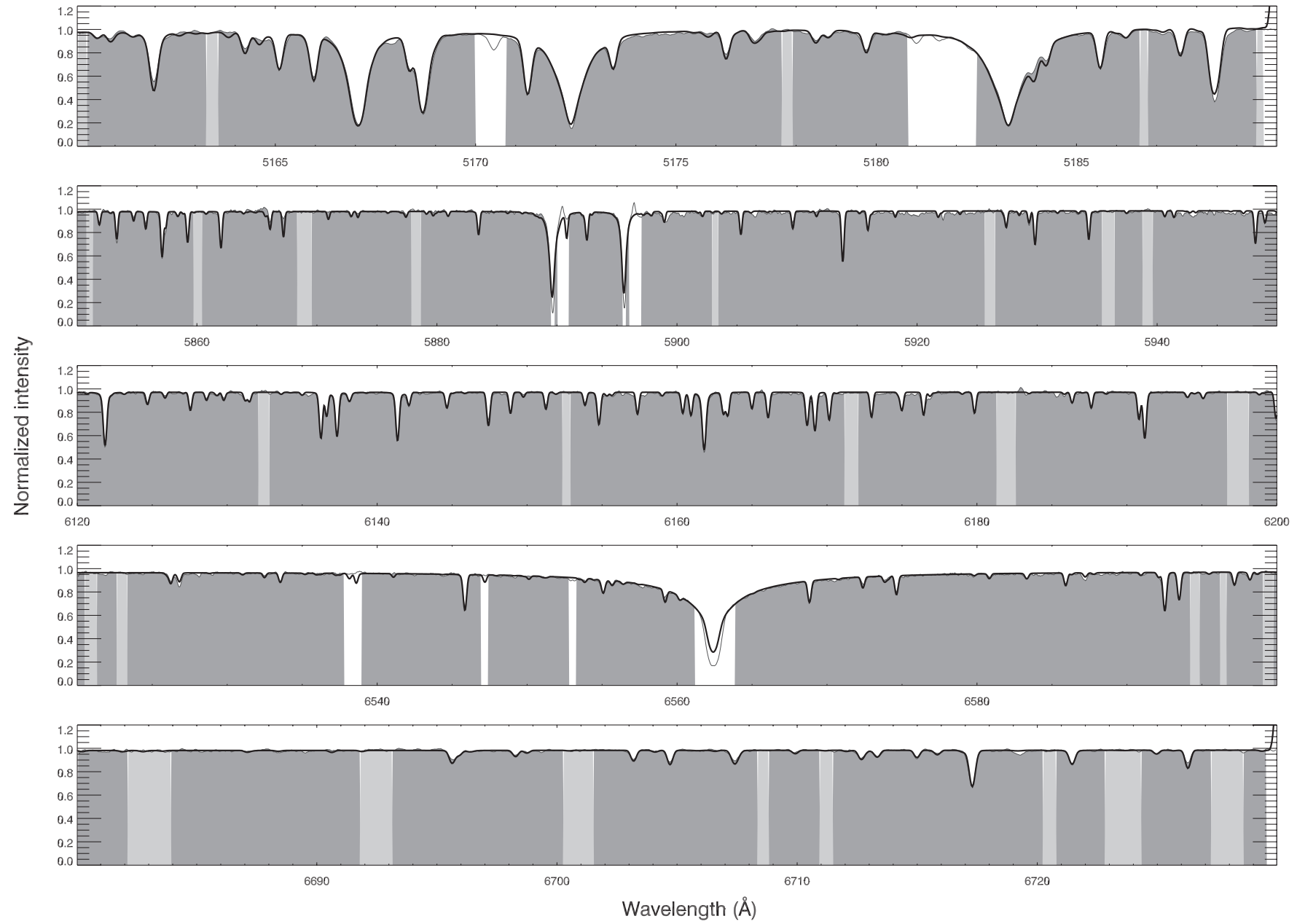

Figure 6. NOT spectrum overlaid with a synthetic spectrum fit during analysis with SME.

Randich 2005) apparent from the weak Li I $(\lambda=678 \mathrm{~nm})$ line, which implied an age of at least $1 \mathrm{Gyr}$.

\subsection{Planetary System Parameters}

We used an updated version of the Markov-chain Monte Carlo (MCMC) fitting procedure described by Collier Cameron et al. (2006) and Pollacco et al. (2008) to determine the full set of system parameters by simultaneously fitting the transit photometry and RV curves. Limb-darkening parameters appropriate to the filter of each of the separate photometric data sets were included, based on the tables of Claret $(2000,2004)$.

The current version of the MCMC code computes the stellar mass as a function of $T_{\text {eff }}, \log \rho_{*}$, and $[\mathrm{Fe} / \mathrm{H}]$ using the procedure described by Enoch et al. (2010), based on the compilation of eclipsing-binary data by Torres et al. (2010). In the analysis for WASP-24, the metallicity $[\mathrm{M} / \mathrm{H}]$ used was derived from a set of spectral features, including those of iron, according to the procedure described in Stempels et al. (2007). The MCMC code uses $T_{\text {eff }}$ and $[\mathrm{M} / \mathrm{H}]$ as MCMC jump variables, constrained by Bayesian priors based on the spectroscopically determined values given in Table 3 .

At each step in the chain, the stellar density was computed from the parameters describing the transit geometry. The stellar mass was then computed using the empirical calibration and the median of the posterior probability distribution for the stellar mass is given in Table 4. RV observations are known to produce a bias toward small but non-zero eccentricities (Lucy \& Sweeney 1971) if the photometric data do not adequately define the ingress and egress, so any measured eccentricity should be considered to be an upper limit. To explore this, we performed multiple runs of the fitting procedure, alternately fixing the eccentricity to zero and allowing it to float, as well as with and without a requirement that the star be on the main sequence. This constraint was applied by deriving, at each step in the chain, the expected main-sequence radius from the power-law approximation of Tingley \& Sackett (2005): $R / R_{\odot}=\left(M / M_{\odot}\right)^{0.8}$. The difference between this value and the stellar radius being used to fit the transit profile at each step is then used in a Gaussian prior. This pulls the stellar radius toward the expected main-sequence value when the quality of the photometry is insufficient to determine the impact parameter precisely.

The star and planetary mass and radii were consistent within the formal errors between the MCMC results with floating and fixed eccentricity, both with and without the main-sequence constraint. We therefore present in Table 4 the system parameters for the $e=0$ case, with the star constrained to the main sequence. The appropriate model RV and light curves are superimposed on the data sets shown in Figures 1-3.

Table 5 compares the mid-times of transit predicted from the ephemeris produced by the MCMC fit with those measured from the data. The times were measured using a minimum-chisquared approach by fitting a model light curve derived from 
Table 4

System Parameters for WASP-24, Derived from the MCMC-fitting Procedure Described in Section 3.2

\begin{tabular}{lccc}
\hline \hline \multicolumn{1}{c}{ Parameter } & Symbol & Value & Units \\
\hline Transit epoch (HJD) & $T_{0}$ & $2455081.37941_{-0.00016}^{+0.00017}$ & $\ldots$ \\
Orbital period & $P$ & $2.34121242 \pm 0.000002$ & days \\
Ratio planet/star area & $\left(R_{p} / R_{s}\right)^{2}$ & $0.01008 \pm 0.00012$ & $\ldots$ \\
Transit width & $\delta \mathrm{t}$ & $0.111844728 \pm 0.0007$ & days \\
Impact parameter & $b$ & $0.653_{-0.019}^{+0.018}$ & $R_{*}$ \\
Stellar reflex velocity & $K$ & $0.1452_{-0.0044}^{+0.0045}$ & $\mathrm{~km} \mathrm{~s}^{-1}$ \\
Center-of-mass velocity & $\gamma$ & $-17.8993 \pm 0.0011$ & $\mathrm{~km} \mathrm{~s}^{-1}$ \\
Orbital eccentricity & $e$ & $0($ adopted $)$ & $\ldots$ \\
Orbital inclination & $i$ & $83.64 \pm 0.31$ & $\mathrm{deg}$ \\
Stellar density & $\rho_{*}$ & $0.502 \pm 0.03$ & $\rho_{\odot}$ \\
Stellar mass & $M_{*}$ & $1.184 \pm 0.027$ & $M_{\odot}$ \\
Stellar radius & $R_{*}$ & $1.331 \pm 0.032$ & $R_{\odot}$ \\
Orbital separation & $a$ & $0.03651 \pm 0.00028$ & $\mathrm{AU}$ \\
Planet radius & $R_{P}$ & $1.3_{-0.038}^{+0.039}$ & $R_{\text {Jup }}$ \\
Planet mass & $M_{P}$ & $1.071_{-0.038}^{+0.036}$ & $M_{\mathrm{Jup}}$ \\
Stellar surface gravity & $\log g_{*}$ & $4.263 \pm 0.017$ & $\ldots$ \\
Planet surface gravity & $\log g_{P}$ & $3.16_{-0.025}^{+0.026}$ & $\ldots$ \\
Planet density & $\rho_{P}$ & $0.487_{-0.040}^{+0.043}$ & $\rho_{\mathrm{Jup}}$ \\
$L_{\text {total }} / L_{\text {critical }}$ & $L_{\text {tot }} / L_{c}$ & $0.4787_{-0.0062}^{+0.007}$ & $\ldots$ \\
Planet equil. temp. (A $=0)$ & $T_{P}$ & $1801.0_{-35.0}^{+37.0}$ & $\mathrm{~K}$ \\
\hline & & & \\
\hline
\end{tabular}

Table 5

Measured and Predicted Times of Transit of WASP-24 b

\begin{tabular}{lccr}
\hline \hline UT date & Measured Tmid (HJD) & Predicted Tmid (HJD) & $\Delta T_{0}(\mathrm{~s})$ \\
\hline 2009 Apr 23 & $2454945.58926_{-0.0002}^{+0.00011}$ & 2454945.58909 & 14.9 \\
2009 May 17 & $2454969.00416_{-0.00055}^{+0.00055}$ & 2454969.00121 & 254.5 \\
2009 Jun 12 & $2454994.75246_{-0.00057}^{+0.0006}$ & 2454994.75455 & -180.2 \\
2009 Jul 17 & $2455029.87085_{-0.00075}^{+0.00075}$ & 2455029.87274 & -162.8 \\
2010 Feb 22 & $2455249.94627_{-0.00064}^{+0.00037}$ & 2455249.9467 & -37.8 \\
2010 Apr 26 & $2455313.15976_{-0.00066}^{+0.000075}$ & 2455313.15944 & 27.3 \\
2010 May 01 & $2455317.84261_{-0.00063}^{+0.00055}$ & 2455317.84186 & 64.6 \\
2010 May 15 & $2455331.8895_{-0.00057}^{+0.00038}$ & 2455331.88914 & 31.3 \\
\hline
\end{tabular}

the system parameters in Table 4 to each data set folded on the determined period and systematically incrementing epoch. While this approach is not model independent, and does not allow an independent determination of the transit duration, it can fit data sets missing data during ingress or egress. The well-measured events are consistent with the predicted times of transit; discrepancies are seen only in data sets where red noise is significant, or where data gaps coincide with ingress/egress.

\subsection{Stellar Age}

In order to constrain the age of this star, we used the theoretical isochrones of Girardi et al. (2000), for the measured metallicity of WASP-24, $Z=Z_{\odot} 10^{[\mathrm{M} / \mathrm{H}]}=0.0223$, following the method of Hebb et al. (2008). We computed the value $R_{*} /\left(M_{*}^{1 / 3}\right)$ as a proxy for stellar density, and plotted it against $T_{\text {eff }}$ for isochrones in the range log age $=6.6-10.15$. Similarly, we extracted evolutionary mass tracks, plotting $R_{*} /\left(M_{*}^{1 / 3}\right)$ versus $T_{\text {eff }}$ for stars of the same mass at each epoch in the age range above.

The location of WASP-24 relative to these tracks could then be found from the star's spectroscopic $T_{\text {eff }}$ and the mean stellar
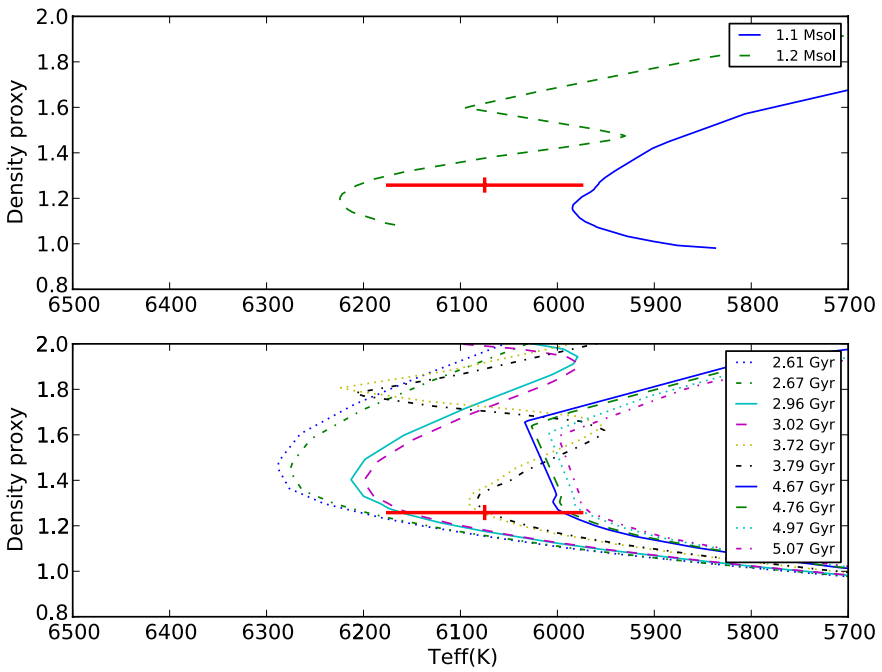

Figure 7. Upper panel: evolutionary mass tracks plotted as density proxy $R_{*} /\left(M_{*}^{1 / 3}\right)$ against effective temperature. Lower panel: isochrones plotted as functions of the same parameters. Both data sets are derived from the theoretical work of Girardi et al. (2000). The position of WASP-24 is indicated with its error ranges.

(A color version of this figure is available in the online journal.)

density, $\rho_{*}=0.502_{-0.03}^{+0.032} \rho_{\odot}$, derived from the MCMC fit. Taking the two isochrones and mass tracks to either side of the star's position (Figure 7), we interpolated along all four to provide a smoothly sampled, denser grid, and then found the values of stellar mass and age from the isochrone and mass track closest to the target's $R_{*} /\left(M_{*}^{1 / 3}\right)$ and $T_{\text {eff. }}$. The process was repeated for the extremes of the $T_{\text {eff }}$ and $\rho_{*}$ error ranges to provide estimates of the uncertainties in the stellar mass and age.

From this analysis, we estimate the age of WASP-24 to be $3.8_{-1.2}^{+1.3} \mathrm{Gyr}$ and the mass to be $1.15_{-0.04}^{+0.05} M_{\odot}$, consistent with the result of the MCMC fitting, as expected. These and other basic parameters of this host star are summarized in Table 3.

\section{DISCUSSION}

Our best fit to the combined RV and photometry data indicate that WASP-24 hosts a $1.071 M_{\text {Jup }}$ planet in a circular orbit with a semimajor axis, $a$, of $0.03651 \mathrm{AU}$. With a radius of $1.3 R_{\mathrm{Jup}}$, WASP-24 b lies in the middle of the main clump of objects on the planetary mass-radius diagram. We compared the measured radius to the theoretical predictions in Fortney et al. (2007). Their paper offers predicted radii for planets of various masses, heavy element fraction (or core mass), orbital separation, and age. We compared Fortney et al.'s (2007) predicted radii for a $1.0 M_{\text {Jup }}$ planet with an orbital separation of $0.02 \mathrm{AU}$ for $1 \mathrm{Gyr}$ and 4.5 Gyr old systems. They predict a radius of $1.179 R_{\mathrm{Jup}}$ for a planet with no core at age $4.5 \mathrm{Gyr}$, and a radius of $1.258 R_{\mathrm{Jup}}$ for the same planet at age $1 \mathrm{Gyr}$. WASP-24 b's radius, $1.3 R_{\mathrm{Jup}}$, actually slightly exceeds the theoretical radius at $1 \mathrm{Gyr}$, although the system is probably close to the $4.5 \mathrm{Gyr}$ old bracket. All other factors being equal, planetary radii decrease with increasing core mass, so these results argue for a small or non-existent planetary core. Given the comparatively low metallicity of WASP-24, a small core would be consistent with the proposed positive correlation suggested by Burrows et al. (2007), between planet core mass and their host star's metallicity. However, detailed customized modeling of the system is required before any firm conclusions can be drawn, as the planetary radius is 
dependent on the body's evolutionary history, irradiation, tidal interactions, the composition of the atmosphere, and efficiency of heat transport within it (Guillot \& Gautier 2009; Burrows et al. 2009).

WASP-24 b may be compared with the well-studied transiting planet HD 189733 b, with a similar orbital period, mass, and radius, though with a more massive host star. As a result, the equilibrium temperature of WASP-24 b, $1801 \mathrm{~K}$, is higher even than HD 189733 b's dayside (1212 K; Knutson et al. 2007). HAT-P-5 b has similar physical characteristics to WASP $24 \mathrm{~b}$ with $M_{\mathrm{HAT}-P-5 b}=1.06 M_{\text {Jup }}$ and $R_{\mathrm{HAT}-P-5 b}=1.26 R_{\mathrm{Jup}}$, and suffers comparable irradiation from its $\sim \mathrm{F} 8$ host star $(P=$ 2.79 days). The systems are also similar in age $\left(\right.$ Age $_{\text {HAT }-P-5}=$ $2.6 \mathrm{Gyr})$, though WASP-24's metallicity $\left(\left[\frac{\mathrm{M}}{\mathrm{H}}\right]=+0.07\right)$ suggests that it has a lower iron fraction $\left(\left[\frac{\mathrm{Fe}}{\mathrm{H}}\right]_{\text {HAT }-P-5}=0.24\right)$.

This work was based on observations made with the Nordic Optical Telescope, operated on the island of La Palma jointly by Denmark, Finland, Iceland, Norway, and Sweden, in the Spanish Observatorio del Roque de los Muchachos of the Instituto de Astrofísica de Canarias. This work made use of the Exoplanet Encyclopedia, http://exoplanet.eu. We are grateful for the constructive suggestions of the anonymous referee.

Facilities: FLWO:2MASS, NOT, Liverpool:2m, FTN, FTS, Euler1.2m, LCOGT

\section{REFERENCES}

Allan, A. 2004, in ASP Conf. Ser. 347, Astronomical Data Analysis Software and Systems XIV, ed. P. Shopbell, M. Britton, \& R. Ebert (San Francisco, CA: ASP), 370

Anderson, D., et al. 2010, ApJ, 709, 159
Burrows, A., \& Orton, G. 2009, in Space Science Ser., Exoplanets, ed. S. Seager (Tucson, AZ: Univ. Arizona Press), in press (arXiv:0910.0248)

Burrows, A., et al. 2007, ApJ, 661, 502

Claret, A. 2000, A\&A, 363, 1081

Claret, A. 2004, A\&A, 428, 1001

Collier Cameron, A., et al. 2006, MNRAS, 373, 799

Collier Cameron, A., et al. 2007, MNRAS, 380, 1230

Cutri, R. M., et al. 2003, 2MASS All-Sky Catalog of Point Sources, NASA/IPAC Infrared Science Archive, http://irsa.ipac.caltech.edu/applications/Gator/

Désert, J.-M., et al. 2009, ApJ, 699, 478

Enoch, R., et al. 2010, A\&A, 516, 33

Fortney, J. J., Marley, M. S., \& Barnes, J. W. 2007, ApJ, 659, 1661

Fortney, J. J., et al. 2008, ApJ, 678, 1419

Girardi, L. 2002, A\&A, 391, 195

Gray, D. F. 1992, The Observation and Analysis of Stellar Photospheres (2nd ed.; Cambridge: Cambridge Univ. Press)

Guillot, T., \& Gautier, D. 2009, in Treatise on Geophysics, Vol. 10: Planets and Moons, ed. G. Shubert \& T. Spohn (Amsterdam: Elsevier), 439

Guillot, T., et al. 2006, A\&A, 453, L21

Hebb, L., et al. 2008, ApJ, 693, 1920

Hebb, L., et al. 2010, ApJ, 708, 224

Iro, N., \& Deming, L. D. 2010, in Proc. IAU Symp. 253, Transiting Planets, ed. F. Pont, D. D. Sasselov, \& M. J. Holman (Cambridge: Cambridge Univ. Press)

Jackson, B., et al. 2009, ApJ, 698, 1357

Knutson, H., et al. 2007, Nature, 447, 183

Knutson, H., et al. 2009, ApJ, 691, 866

Latham, D., et al. 2010, ApJ, 713, 140

Lucy, L. B., \& Sweeney, M. A. 1971, AJ, 76, 544

Pollacco, D., et al. 2006, PASP, 118, 1407

Pollacco, D., et al. 2008, MNRAS, 385, 1576

Queloz, D., et al. 2001, A\&A, 379, 279

Santos, N. C., et al. 2002, A\&A, 392, 215

Sestito, P., \& Randich, S. 2005, A\&A, 442, 615

Stempels, E., et al. 2007, MNRAS, 379, 773

Tingley, B., \& Sackett, P. 2005, ApJ, 627, 1011

Torres, G., Andersen, J., \& Giménez, A. 2010, A\&AR, 18, 67

Valenti, J. A., \& Piskunov, N. 1996, A\&AS, 118, 595 